RAD Conference Proceedings, vol. 4, pp. 185-189, 2020

ISSN 2466-4626 (online) | DOI: 10.21175/RadProc.2020.37

www.rad-proceedings.org

\title{
PROTON BORON CAPTURE THERAPY: DOSE CALCULATIONS AND A POSSIBLE NEW MEASUREMENT
}

\author{
M. Mastromarco' ${ }^{*}$, A. Digennaro',2, A. Mazzone3, \\ P. Finocchiaro4, J. Praena5, I. Porras5, N. Colonna ${ }^{1}$ \\ ${ }^{1}$ Istituto Nazionale di Fisica Nucleare (INFN), Sez. di Bari, Bari, Italy \\ ${ }^{2}$ Università degli Studi di Bari (UniBa), Dipartimento di Fisica “M. Merlin”, Bari, Italy \\ ${ }^{3}$ Consiglio Nazionale delle Ricerche, Istituto di Cristallografia (CNR - IC), Bari, Italy \\ 4Istituto Nazionale Fisica Nucleare, Laboratori Nazionali del Sud (INFN - LNS), Catania, Italy \\ 5Departamento de Física Atómica, Molecular y Nuclear, Universidad de Granada, Granada, Spain
}

\begin{abstract}
Since a few decades, proton therapy is being widely used for cancer treatment, with minimal dose to healthy tissues and surrounding organs. Recent studies have suggested that the efficacy of proton therapy could be enhanced if natural boron is selectively accumulated in the tumor tissues. Such an increase is attributed to the protonboron fusion reaction that leads to the production of low-energy a-particles ( 2.9 MeV), a mechanism that resembles the well-known Boron Neutron Capture Therapy. However, analytical calculations and detailed Monte Carlo simulations with GEANT4, both in a macro- and micro-dosimetry approach, indicate that the effect of the $p+{ }^{11} B \rightarrow 3 \alpha$ reaction, at the standard Boron concentration levels (less than 100 ppm), is orders of magnitude lower than the one of the primary proton beam inside the tissues. In an attempt to solve this discrepancy, an experimental campaign will be carried out with a low-energy proton beam at the CNA laboratory, in Seville. In this talk, we present the latest results of detailed calculations and Geant4 simulations of the dose related to PBCT and describe the concept at the basis of the proposed experimental activity on the PBCT efficacy.
\end{abstract}

Key words: Proton Therapy, PBCT, GEANT4, Monte Carlo simulations

\section{INTRODUCTION}

Nowadays, proton therapy is one of the most technologically advanced methods to deliver radiation treatments to the deep-seated tumors; this technique takes advantage of the increase of deposited energy at the end of the proton range, the so-called Bragg peak. In comparison with the standard radiation therapy, this process allows to deliver curative radiation doses to the tumors while reducing doses to healthy tissues and side effects.

Recent studies based on Monte Carlo simulations for radiation transport [2] argued that a factor of two is gained on the delivered dose by proton beams if boron is accumulated in the tumor tissues, attributing such effect to the proton-boron fusion reaction, $\mathrm{p}+{ }^{11} \mathrm{~B} \rightarrow 3 \alpha$. A more recent experimental study performed by Cirrone et al. [1], indicated that the dose delivered in proton therapy is enhanced if adequate concentration of natural boron is added to the tumor cells prior to proton irradiation; in analogy to the well-known Boron Neutron Capture Therapy, the authors have referred to this mechanism as "Proton Boron Capture Therapy" (PBCT). By irradiating samples of prostatic tumor cells with and without boron carrier (sodium borocaptate, $\mathrm{Na}_{2} \mathrm{~B}_{12} \mathrm{H}_{11} \mathrm{SH}$ or $\mathrm{BSH}$ ), the authors of Ref. [1] have shown that the tumor cells survival probability undergoes a strong reduction.

Although the effect of BSH on the probability of cell survival is quite clear, the claim that this effect is related to the proton boron fusion reaction is not supported by dose calculations. The aim of this contribution is to shed light on the potential role of the $\mathrm{p}+{ }^{11} \mathrm{~B} \rightarrow 3 \alpha$ reaction by showing results obtained from reaction rate calculations, either in macro- or microdosimetry approach and by realistic Monte Carlo simulations of the corresponding delivered dose.

\section{DELIVERED Dose}

With the aim of verifying the effective role of the reaction $\mathrm{p}+{ }^{11} \mathrm{~B} \rightarrow 3 \alpha$ and hence of the proposed Proton Boron Capture Therapy (PBCT), we have performed analytical calculations of the corresponding delivered dose, as well as realistic Monte Carlo simulations with the specifically optimized GEANT4 toolkit.

\subsection{Dose Calculations}

According to Cirrone et al. [1], the maximum effect of PBCT is obtained at the end of proton range, due to the presence of a resonance like-structure ( 1 barn @ $700 \mathrm{keV}$ [3]).

*mario.mastromarco@ba.infn.it 
Starting from the value of the reaction cross section, it is possible to estimate the reaction yield, and hence, the delivered dose. By using the maximum cross section value ( $\sim$ barn), so assuming a flat behavior, one estimates the delivered dose in the best condition scenario, as any other assumption would lead to a lower reaction rate.

In order to calculate the reaction yield (i.e. the probability that a proton undergoes a reaction), one can consider the region of the Bragg peak, in particular a slab of tissue of $1 \mathrm{~mm}$ thickness at the end of the proton range, where protons release in average 10 $\mathrm{MeV}$. Assuming a BSH concentration of $80 \mathrm{ppm}$, a value of the reaction yield lower than $10^{-6}$ is obtained, meaning that only one proton per million reacts with ${ }^{11} \mathrm{~B}$, producing three alpha-particles. Since both protons and the three $\alpha$-particles emitted in the reaction release approximately $10 \mathrm{MeV}$ in the slab, this yield corresponds also to the ratio between the dose produced by the PBCT, and the one produces by the primary proton beam. Therefore, it seems rather unlikely that the $\mathrm{p}+{ }^{11} \mathrm{~B} \rightarrow 3 \alpha$ reaction might enhance proton therapy.

Another important consideration is related to the large presence of water in biological tissues. In fact, proton-induced reaction on Oxygen, leads also to $\alpha$ particles emission with a cross section $\sim 100 \mathrm{mb}$ Ref. $[4,5]$. Although this is a threshold-reaction for proton energies above $5.5 \mathrm{MeV}$, one can expect a contribution to the total dose growth much higher than the one obtained by $\mathrm{p}+{ }^{11} \mathrm{~B} \rightarrow 3 \alpha$ with a quite flat dose distribution over the whole proton range. Calculations, performed assuming a proton beam of $62.5 \mathrm{MeV}$ impinging on a $3.5 \mathrm{~cm}$ deep water phantom loaded with $\mathrm{BSH}$ at a concentration of $80 \mathrm{ppm}$, show that indeed this is the case, as demonstrated in Figure 1.

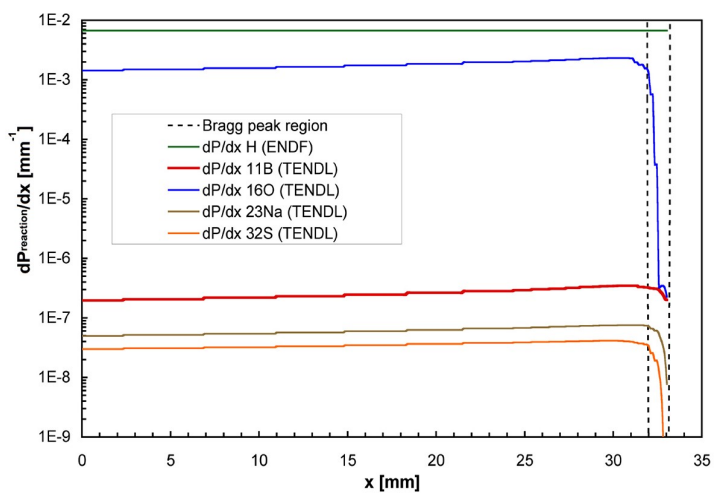

Figure 1 . The probability per unit length of proton interaction as a function of depth inside water, for an incident proton of $62.5 \mathrm{MeV}$. The different curves represent reactions on the different elements, either phantom (or water) or BSH (80 ppm).

Figure 2 shows a more quantitative assessment on the delivered dose by the three $\alpha$-particles from $\mathrm{p}+{ }^{11} \mathrm{~B} \rightarrow 3 \alpha$ (PBCT) and the dose delivered by the primary proton beam; calculations here take into account the Q-value of the reaction and the proton kinetic energy transferred to the a-particles. Apart from being in absolute very low, the ratio between the dose from PBCT and the one due to the primary proton, as a function of depth, is opposite to what would be desirable for therapeutic effectiveness of proton therapy and this is clearly in contradiction with results reported in Ref. [1]

As the macroscopic dose calculation may not be very accurate in predicting the damage at the cellular level, we have also considered a micro/nano dosimetric approach. While a proper microdosimetric approach, as described in the ICRU report 36, would be the one used for example in Ref. [13], one can assume that boron preferentially accumulates in the cell nucleus, and that the produced $\alpha$-particle can efficiently deliver a lethal dose to the cells.

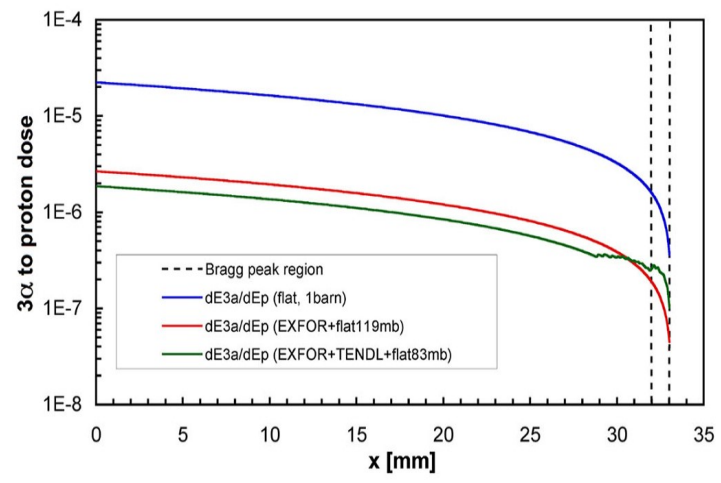

Figure 2. Ratio of proton boron capture to primary dose, as a function of the depth inside phantom (water).

In this case, one can estimate the cell survival probability, by calculating the number of $\alpha$-particles produced by the $\mathrm{p}+{ }^{11} \mathrm{~B}$ reaction per unit volume for a given proton dose in the Bragg peak. It turns out that at $4 \mathrm{~Gy}$ of delivered proton dose (corresponding to a proton fluence of $2.5 \times 10^{8}$ protons $/ \mathrm{cm}^{2}$ ), approximately $10^{4} \mathrm{\alpha}$-particles $/ \mathrm{cm}^{3}$ are produced by proton boron capture in the irradiation. In the assumption that each a-particle kills the cancer cell, by assuming $10^{8}$ cells $/ \mathrm{cm}^{3}$ of tissue, the resulting decrease in survival fraction is as small as $0.01 \%$, i.e. at least three order of magnitude lower than the one reported in Ref. [1]. Therefore, even the micro/nano dosimetry approach leads to the conclusion that the reaction $\mathrm{p}+{ }^{11} \mathrm{~B} \rightarrow 3 \alpha$ cannot be claimed as the responsible in the cell survival probability.

\subsection{GEANT4 Simulations}

Monte Carlo simulations in proton therapy are typically performed with three main tools: MCNP [6], FLUKA [7] and GEANT4 [8]; apart from small differences in hadronic processes Ref. [9, 10], general features of the dose profile are well reproduced by the different codes.

Monte Carlo simulations can also be used to estimate the dose related to a specific reaction, in particular the one at the basis of the Proton Boron Capture Therapy. To this purpose, we have used the toolkit GEANT4. Although such code has been 
generated for high-energy physics simulations, recent developments have been made to improve the hadronic interactions at very low energy through the so-called $Q G S P$-BIC-ALLHP model.

The reliability of Monte Carlo simulations of the dose in boron-enhanced proton therapy depends on the use of an accurate reaction cross section. We have verified that the cross section used in GEANT4 within the QSGSP-BIC-ALLHP model agrees (within a factor of two) with the experimental one, in the region where they overlap; simulations permit also to verify other important aspects like the presence of additional reactions that in some way could contribute to the decrease of the survival probability reported in Ref. [1].

In the code, a volume of $2 \times 2 \times 10 \mathrm{~cm}^{3}$ of water has been implemented as a software replica of the phantom; a pencil beam of $10^{7}$ primary protons, perpendicular to the phantom input face and with 2 mm Gaussian spatial distribution has been generated, with an energy distribution suitably constructed in order to obtain a Spread Out Bragg Peak (SOBP) as close as possible to the one of proton therapy facility of INFN-Laboratori Nazionali del Sud, shown in Ref. [1].

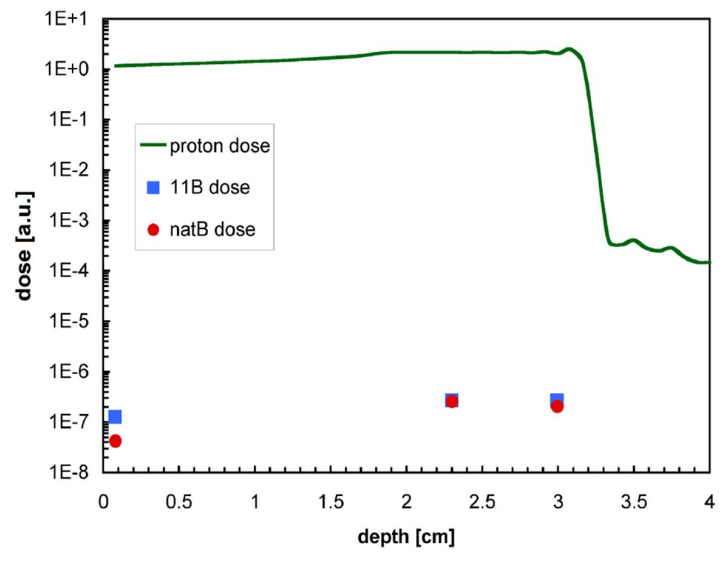

Figure 3. The depth profile of protons in the phantom, with energy distribution so to reproduce the SOBP of Ref. [1]

(green curve), compared with the dose related to $\mathrm{p}+{ }^{11} \mathrm{~B} \rightarrow \mathrm{a}$ reaction, in $1 \mathrm{~mm}$ slabs along the beam direction, loaded with $80 \mathrm{ppm}$ of nat $\mathrm{B}$ or pure ${ }^{11} \mathrm{~B}$.

In the simulations, we have reproduced the geometry and other features of the experiment reported in Ref. [1]. In particular, we have determined the dose produced by the $\mathrm{n}+{ }^{11} \mathrm{~B}$ reaction in $1 \mathrm{~mm}$ thick water slabs at the entrance of the proton beam at 0.2 $\mathrm{cm}(\mathrm{P} 1)$ and in two position of SOBP at $2.4 \mathrm{~cm}(\mathrm{P} 2)$ and $3.0 \mathrm{~cm}\left(\mathrm{P}_{3}\right)$. In each of these slabs, a concentration of $80 \mathrm{ppm}$ of $\mathrm{BSH}$ with natural boron was included. The number of $\alpha$-particles emitted in the slabs was compared with the one obtained without $\mathrm{BSH}$; simulations took into account all possible protoninduced reactions responsible for $\alpha$-particles production. Finally, simulations were also performed with $100 \%$ of ${ }^{11} \mathrm{~B}$ and ${ }^{10} \mathrm{~B}$, respectively.

To considerably reduce the time needed to run the simulations, the concentration was increased of three order of magnitude relative to the realistic case, i.e. a BSH concentration of $8 \%$; results then were scaled down by the same gain factor. During the simulations, the $\alpha$-particles produced in the $1 \mathrm{~mm}$ slabs loaded with $8 \%$ of nat $\mathrm{B},{ }^{11} \mathrm{~B}$ and ${ }^{10} \mathrm{~B}$ at three different positions inside the phantom (at the entrance and in the SOBP), were recorded. The results, affected by an uncertainty of a few percent, indicate that the probability of $\alpha$-particles production in proton-induced reactions on boron is of the order of $10^{-7}$. Therefore, simulations as well confirm that the number of proton- or neutron-induced reactions on boron in proton therapy is practically negligible.

This is finally confirmed by the comparison, shown in Figure 3, of the dose produced by the boron capture reaction for $80 \mathrm{ppm}$ of $\mathrm{BSH}$ with nat $\mathrm{B}$ or pure ${ }^{11} \mathrm{~B}$ (solid symbols), with the one produced by the proton beam (curve). Once more this comparison indicates that the PBCT cannot play a role in the proton therapy and a different explanation have to be searched to explain the decrease in the cell survival probability reported in Ref. [1].

\section{PBCT MEASUREMENT}

A possible mean to verify the simulations results and determine the effectiveness of proton boron capture therapy would be to study it directly in the proton Bragg peak, where the number of $\mathrm{p}+{ }^{11} \mathrm{~B}$ reactions is expected to be the maximum.

To this end, we have designed a measurement with a low-energy proton beam impinging on a thin layer of prostatic tumor cells with a suitable energy so that the Bragg peak falls within the cellular layer. In particular, we have considered the use of an existing beam line dedicated to radiobiology studies at the $3 \mathrm{MV}$ Tandem Accelerator of the Centro Nacional de Acceleradores, (CNA), in Sevilla, Spain. More information on the Tandem and on the proton beam line can be found in Ref. [11].

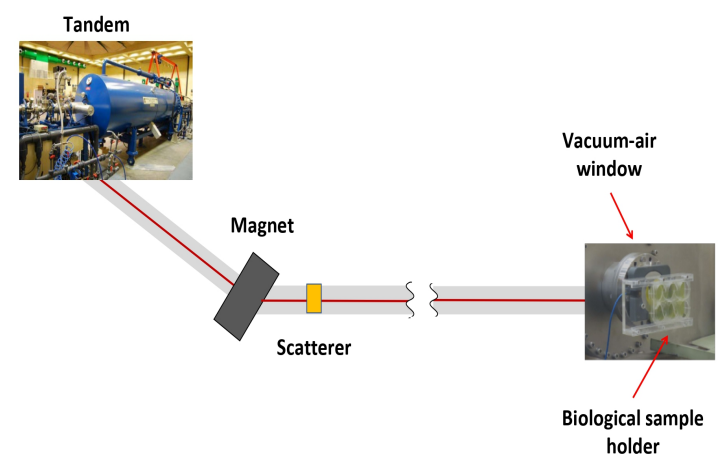

Figure 4. The proton beam line used for radiobiology studies at the $3 \mathrm{MV}$ Pelletron Tandem accelerator at CNA, Seville.

The experimental setup is shown schematically in Figure 4 . The proton beam is produced by the Tandem accelerator with an initial energy of $5.6 \mathrm{MeV}$. A Au foil 
of $2.8 \mu \mathrm{m}$ thickness, positioned $5.2 \mathrm{~m}$ upstream of the cell samples to be irradiated, is used to increase the dimension of the proton beam, so that it impinges on the sample with a practically uniform spatial distribution. A Kapton foil of $50 \mu \mathrm{m}$ thickness acts as the vacuum-air interface, at the exit of the proton beam line. A sample holder, hosting up to 6 samples, is mounted in air, just outside the proton beam line, $1 \mathrm{~cm}$ far from the proton exit window. It can be controlled remotely so to insert sequentially the samples in the beam without entering the irradiation station. Each sample is made of a mylar foil, acting as backing for the cellular layer, mounted on a square aluminum structure. Since the energy of the proton beam impinging on the sample is still too high, the mylar foil can also be used to suitably degrade it so that the Bragg peak of the proton beam falls exactly within the cellular layer, thus maximizing the number of possible protoninduced reactions on ${ }^{11} \mathrm{~B}$. To this end, the optimal thickness of the foil has to be calculated. Both the TRIM code [12] and the GEANT4 toolkit indicate that for the proton beam here described, the mylar foil has to be $250 \mu \mathrm{m}$ thick. The results of the energy loss calculations are shown in Figure 5.

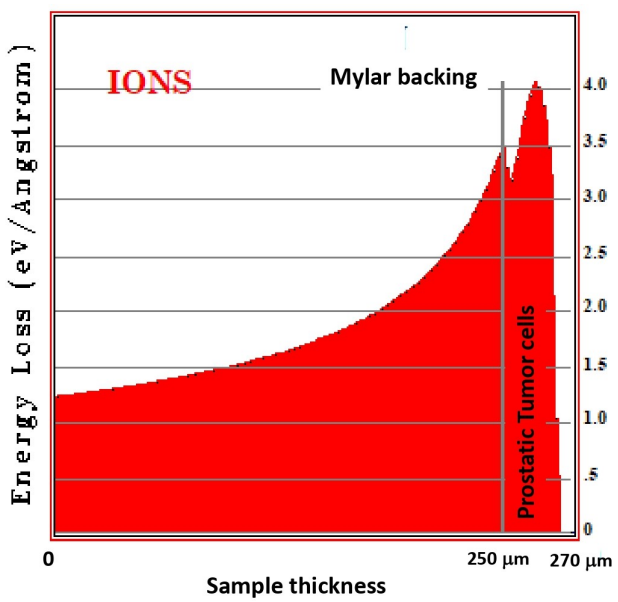

Figure 5. Energy loss by the proton beam in the mylar backing and in the prostatic cell layer. For a suitable choice of the thickness of the mylar backing (in this case $250 \mu \mathrm{m}$ ), a Bragg peak is formed by the impinging proton beam within the cellular layer.

In order to produce a thin and uniform layer of prostatic cells on the mylar backing, the sample is filled with a culture medium containing the cells, and placed horizontally, so to fall on the foil (by gravity), and adhere to it. With this process, a uniform cellular layer of thickness between 15 and $20 \mu \mathrm{m}$ can be produced. Following this step, the sample can be mounted on the beam line vertically, and with the culture medium still contained in it, so to ensure stable conditions to the cells.

The measurement here described was planned and ready to be performed in the first half of 2020 but had to be postponed due to the pandemic situation. It will however be performed as soon as circumstances will allow.

\section{CONCLUSION}

At the declared boron concentration, both calculations and simulations lead to the same conclusions: the reaction probability of PBCT is too low to bear any effect in proton therapy at the commonly used BSH concentration.

In order to experimentally verify the results of the simulations, we propose to perform a measurement with low-energy proton beams directly on a layer of prostatic tumor cells. The measurement here described has already been planned and will be performed as soon as circumstances will allow.

\section{REFERENCES}

1. G.A.P. Cirrone et al., "First experimental proof of Proton Boron Capture Therapy (PBCT) to enhance proton therapy effectiveness", Sci. Rep., vol. 8, article no. 1141, 2018. https://doi.org/10.1038/s41598-018-19258-5

2. D.-K. Yoon, J.-Y. Jung, T. S. Suh, "Application of proton boron fusion reaction to radiation therapy: A Monte Carlo simulation study", Appl. Phys. Lett., vol. 105, no. 22, article no. 223507, 2014. https://doi.org/10.1063/1.4903345

3. R.E. Segel, S.S. Hanna, R.G. Allas, "States in $\mathrm{C}^{12}$ between 16.4 and 19.6 MeV", Phys. Rev., vol. 139, no. B4, article no. B818, 1965 . https://doi.org/10.1103/PhysRev.139.B818

4. V.V. Zerkina, B. Pritychenko, "The experimental nuclear reaction data (EXFOR): Extended computer database and web retrieval system", Nucl. Instr. Meth. Phys., Research Section A, vol. 888, pp. 31-43, 2018. https://doi.org/10.1016/j.nima.2018.01.045

5. W. Gruhle, B. Kober "The reactions ${ }^{16} \mathrm{O}(\mathrm{p}, \alpha),{ }^{20} \mathrm{Ne}(\mathrm{p}, \alpha)$

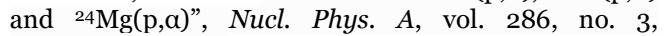
pp. 523-530, 1977. https://doi.org/10.1016/0375-9474(77)90601-7

6. L.S. Waters, “MCNPX 2.6.o User's Guide", Los Alamos National Laboratory, 2008.

7. G. Battistoni et al., "The FLUKA code and its use in hadron therapy", Il Nuovo Cimento C, vol. 31, no. 1, pp. 69-75, 2008. https://doi.org/10.1393/ncc/i2008-10281-9

8. S. Agostinelli et al., "GEANT4-a simulation toolkit", Nucl. Instr. Meth. Phys., Research Section A, vol. 506, no. 3, pp. 250-303, 2003. https://doi.org/10.1016/So168-9002(03)01368-8

9. U. Titt, B. Bednarz, H. Paganetti, "Comparison of MCNPX and Geant4 proton energy deposition prediction for clinical use", Phys. Med. Biol., vol. 57, no. 20, pp. 6381-6393, 2012. https://doi.org/10.1088/0031-9155/57/20/6381

10. C. Robert et al., "Distribution of secondary particles in proton and carbon-ion therapy: a comparison between GATE/Geant4 and FLUKA Monte Carlo codes", Phys. Med. Biol., vol. 58, no. 9, pp. 2879-2900, 2013. https://doi.org/10.1088/0031-9155/58/9/2879

11. M. C. Battaglia, "Dosimetry studies for radiation therapy with photons and radiobiology using lowenergy protons: 1) 2D dose verification for complex radiotherapy treatments, 2) Dosimetry of low-energy protons and first radiobiological experiment”, Ph.D. dissertation, University of Seville, Spain, 2017.

Retrieved from:

https://digital.csic.es/bitstream/10261/196356/1/Dosi metry studies.pdf

Retrieved on: Aug. 15, 2020 
M. Mastromarco et al., Proton boron capture therapy..., RAD Conf. Proc., vol. 4, 2020, 185-189

12. W. Möller, W. Eckstein, "Trydin - A TRIM simulation code including dynamic composition changes", Nucl. Instr. Meth. Phys., Research Section B, vol. 2, no. 1-3, pp. 814-818, 1984.

https://doi.org/10.1016/0168-583X(84)90321-5

13. T. Sato, Sh.-i. Masunaga, H. Kumada, N. Hamada, "Microdosimetric modeling of biological effectiveness for Boron Neutron Capture Therapy considering intraand intercellular heterogeneity in ${ }^{10} \mathrm{~B}$ distribution", Sci. Rep., vol. 8, article no. 988, 2018.

https://doi.org/10.1038/s41598-017-18871-o 